\title{
INDIVIDUAL DIFFERENCES IN THE EXCITATION TRANSFER EFFECT
}

\author{
Scott C. BunCe, Randy J. Larsen* and Maribel CruZ \\ Department of Psychology, University of Michigan, 580 Union Drive, Ann Arbor, MI 48109-1346, U.S.A.
}

(Received 10 December 1992)

\begin{abstract}
Summary-Excitation transfer refers to the main effect of an arousing experience (e.g. exercise) on subsequent emotional reactions (e.g. reactions to emotional photographs). Residual arousal from the earlier task is said to "transfer" to the latter task, with the arousal being misattributed to subsequent stimuli. Studies examining individual differences in excitation transfer have focused on physical characteristics, such as cardiovascular fitness. However, because excitation transfer involves both arousal and hedonic tone, it may be that personality dimensions relevant to arousability or emotional reactivity may predict individual differences in the excitation transfer effect. Several hypotheses were proposed based upon differing personality theories. Results supported the notion that excitation transfer is strongest for the hedonically-valenced emotions with which the person has relatively less experience. Extraversion predicted excitation transfer to unpleasant (but not pleasant) stimuli, and Neuroticism predicted excitation transfer to pleasant (but not unpleasant) stimuli. Results are discussed in terms of processing bodily information more during unfamiliar than familiar affective states.
\end{abstract}

Excitation transfer, first described by Zillman (1971), refers to the influence of a prior episode of arousal on subsequent emotional responses. In the absence of any environmental cues as to the actual source of arousal, residual arousal can be misattributed to a subsequent stimulus. After an initial period of arousal, an individual's skeletal-motor system rapidly adapts, and the person subjectively feels that he or she has returned to a normal level of arousal. Autonomic or cardiovascular adjustment, however, is comparatively sluggish and requires more time to adapt. There consequently exists a time period during which the individual feels 'normal', yet remains at an elevated level of autonomic arousal. It is this lag time in excitatory adjustment, termed residual excitation, that allows for excitation transfer. Lacking environmental cues about the actual source of this residual arousal, it is misattributed to a subsequent stimulus, resulting in an intensification of the individual's experience of the new stimulus.

Excitation transfer has been empirically demonstrated for a number of emotional reactions. For example, residual arousal from sexual excitation can intensify aggression (e.g. Donnerstein \& Hallam, 1978; Zillman, Bryant, Comisky \& Medoff, 1981), arousal transferred from physical exercise can intensify both anger and aggressive behavior (Zillman \& Bryant, 1974; Zillman, Katcher \& Milavsky, 1972), and prosocial behavior can be facilitated by residual sexual arousal (Jaffe, 1981; Mueller \& Donnerstein, 1981).

Few studies have examined individual differences in the propensity for excitation transfer. While Zillman (1983) reports that individual differences exist, all of these studies have focused on differences in physical characteristics, such as aerobic fitness. For example, Cantor, Zillman and Day (1978) report that $S$ s with lower cardiorespiratory fitness showed significantly greater transfer of arousal responses to medical and erotic films. Cox, Evans and Jamieson (1979) demonstrated that $S \mathbf{s}$ with greater aerobic fitness showed a smaller period of residual arousal.

Although these studies focus on individual differences in physical fitness, arousal- or affect-based theories of personality might be used to generate hypotheses about individual differences in the excitation transfer effect. Zillman's theory of excitation transfer suggests that personality may relate to the transfer of excitation in two ways, through either the excitatory or the experiential components of the emotional experience.

Zillman $(1978,1979)$ has suggested that emotional behavior consists of three components, a dispositional, an excitatory, and an experiential component, which combine to produce a phasic

*To whom correspondence should be addressed. 
response to an emotional stimulus. The dispositional component is the initial skeletal-motor behavior associated with a direct response to emotion-inducing stimuli. This component is conceived as a response-guiding system, which operates prior to complex cognitive mediation. The excitatory component, or autonomic arousal, is seen as a response-energizing mechanism. Excitatory reactions are also thought to be directly under stimulus and reinforcement control, without necessary mediation by complex cognitions. The excitatory reactions refer to heightened activity in the sympathetic nervous system which serve the mechanism of fight or flight. According to Zillman, however, this motivation to act does not involve specific teleological aims, as do, for example, hunger and thirst. Rather, the excitatory reaction is independent of particular emotions such as anger or fear, but is dependent upon the response-guiding mechanisms. The third component of emotional behavior is the experiential component, comprised of the conscious experience of either the excitatory or the skeletal-motor reactions, or both.

Personality variables relevant to arousal, reactivity, or attention to bodily states could relate to how a person is influenced by residual arousal via the excitatory or experiential components of the emotion. Personality constructs such as extraversion, neuroticism, augmenting/reducing, or repression might moderate the effects of residual arousal or subsequent emotional reactions. This moderation could occur at either the excitatory level (through individual differences in reactivity to arousing stimuli, e.g. extraversion, reducing/augmenting) and/or at the level of cognitive interpretations of experienced bodily states (through individual differences in the tendency to notice, attend to, and negatively interpret unusual sensations, e.g. neuroticism (Watson \& Pennebaker, 1989).

The arousal theory of extraversion (Eysenck, 1967) can be used to make the prediction that, because extraverts are less arousable than introverts, extraversion scores should be negatively related to the excitation transfer effect, regardless of the hedonic tone of the transfer stimulus. Similar predictions can be made for constructs referring to individual differences in general reactivity. Petrie (1967) for instance, has suggested that persons can be characterized as either sensory augmenters, reducers, or moderates, classifications indicative of their reactivity to sensory stimulation. Augmenters are described as sensitive to sensory stimulation across a variety of modalities. Conversely, reducers tend to be characteristically less reactive to sensory stimuli than either augmenters or moderates. A related individual difference dimension is "strength of the nervous system." This concept is based on Pavlov's definition of the strength of the nervous system as the "working capacity of nerve cells." According to Pavlov, the working capacity of a cell is determined by the maximum amount of work it can perform at one time coupled with the length of time the cell can perform at that level. Augmenters are thought to have relatively "weak" nervous systems and are consequently more sensitive to incoming stimulation, whereas reducers have "strong" nervous systems and are relatively less sensitive to stimulation. Because augmenters are more sensitive to incoming stimulation, they may display relatively more excitation transfer than reducers. Several questionnaire measures of reducing/augmenting have been developed that can readily assess this personality dimension (Herzog, Williams \& Weintraub, 1985; Vando, 1974).

Gray's theory of personality functioning $(1964,1970,1987)$ provides a different model for making predictions. On the basis of neurophysiological research, Gray modified Eysenck's theory of extraversion and neuroticism, proposing two emotion-based neuronal systems that are responsible for behavioral regulation. The Behavioral Activation System (BAS) is believed to regulate behavior in response to signals of reward, while the Behavioral Inhibition System (BIS) regulates behavior by responding to signals of punishment or frustration via non-reward. The BAS and BIS are theoretically orthogonal, with the BAS giving rise to the personality dimensions of Impulsivity and Extraversion, which is linked with sensitivity to signals of reward, and the BIS giving rise to Anxiety and Neuroticism, a predilection to attend to signals of punishment.

The existing theories of arousal or affect-based personality variables, however, offer divergent hypotheses about the relationship between personality and the excitation transfer effect. These hypotheses relate to two aspects of the excitation transfer paradigm, general arousability and hedonic tone.

The first class of hypotheses suggest that individuals who are low on arousability (e.g. extraverts, reducers) should be expected to show diminished effects of residual arousal on subsequent emotional reactions. That is, for persons low in arousability, the induction of residual arousal may 
be less effective or impact less on conscious awareness than for persons who are more arousable. This category of hypotheses would predict negative relations between such personality variables as extraversion and reducing/augmenting and the excitation transfer effect, regardless of the hedonic tone of the arousal transfer stimulus.

A second category of hypotheses concerns the relationship of personality to the hedonic tone of the arousal transfer stimulus. Existing theories would suggest opposing correlations between affect-relevant personality variables and the hedonic tone of the excitation transfer stimulus. A number of studies have linked Extraversion with a predisposition to experience positive affect and Neuroticism with a predisposition to experience negative affect (Costa \& McCrae, 1980; Emmons \& Diener, 1985; Larsen \& Ketelaar, 1989, 1991; O'Malley \& Gillette, 1984; Warr, Barter \& Brownbridge, 1983; Watson \& Pennebaker, 1989). An intuitive and straightforward hypothesis would predict that such affect-specific personality dimensions would influence the degree of excitation transfer depending on the hedonic tone of the transfer stimulus. Zillman's theory would suggest that those individuals who are physiologically more reactive to stimuli of a particular hedonic tone would also be more likely to exhibit excitation transfer in response to those stimuli. Because extraverts are more reactive to positively-toned stimuli, they may transfer excitation more readily than introverts to hedonically-positive stimuli. Similarly, high-neuroticism $S$ s (compared to stable $S$ s) may show relatively more excitation transfer to hedonically-negative stimuli.

There are, however, theoretical reasons for proposing an opposite pattern of results. According to Pennebaker (Pennebaker, 1978), people are more attentive when confronted with stimuli that are less familiar. That is, information processing is more effortful during novel as opposed to typical experiences. During an unfamiliar experience a person will seek out and attend to more sources of information in evaluating that experience. Assuming one's bodily state is a source of information one may use to evaluate emotions, we should expect persons to attend to their bodily state more during the experience of unfamiliar than familiar emotions. Arousal transfer should occur when a person is experiencing an emotion with which they are relatively less experienced. If this is true, then we might expect extraverts to show more arousal transfer than introverts to hedonically-negative stimuli, and high- $\mathrm{N}$ subjects to show more transfer than low-N subjects to hedonically-positive stimuli. Because extraverts are more experienced with positive affect, they will have less need to attend to their bodily reactions when evaluating their response to positive stimuli. Conversely, because they are less experienced with negative affect, extraverts may be more likely to attend to their arousal state when confronted with unpleasant stimuli and hence misattribute residual arousal to those stimuli (i.e. exhibit the excitation transfer effect to negative stimuli). The logic is similar for neuroticism; because high-neuroticism $S$ s are less familiar with positive affect, they may be more likely to attend to their physical state when asked to evaluate pleasant stimuli, and hence misattribute residual arousal to positively toned but not negatively toned stimuli.

In summary, the present study represents an initial examination of personality and individual differences in the excitation transfer effect. While the study is exploratory in nature, there are theoretical reasons for expecting various patterns of results. One theoretical rationale posits a general reactivity effect, such that personality variables associated with lowered general arousability (e.g. extraversion, reducing) are predicted to relate negatively to the magnitude of the excitation transfer effect, regardless of the nature of the transfer stimulus. A second theoretical rationale posits that the hedonic tone of the transfer stimulus will interact with specific personality variables in determining the magnitude of the excitation transfer effect. Personality dimensions associated with positive emotionality (e.g. extraversion) and dimensions associated with negative emotionality (neuroticism, anxiety) might differentially predict arousal transfer to pleasant and unpleasant stimuli, respectively. A third theoretical rationale suggests predictions exactly opposite. Because extraverts are 'experts' in positive affect they may be less likely to misattribute arousal to positive stimuli. Instead, because they have less familiarity with negative affect, extraverts may be more likely to notice and attend to their residual arousal when responding to negative stimuli. The same logic predicts that high-neuroticism persons will show more excitation transfer to positive affect stimuli. These predictions posit a contrast effect, in that the arousal transfer will occur in the hedonic condition with which the person has the least familiarity; negative affect for extraverts, positive affect for neurotics.

Because of competing theoretical views, specific conflicting predictions are made. As described 
above, extraversion and neuroticism may relate to heightened excitation transfer to either negative or positive stimuli, or only arousal-relevant dimensions will relate to arousal transfer. One strength of the present study is that we examine excitation transfer effects to both positive and negative stimuli within the same design. As such, differential excitation transfer effects for hedonically opposite stimuli may be explored.

\section{METHOD}

\section{Subjects}

Forty female undergraduate $S$ s participated in this study in partial fulfillment of class requirements. $S$ s were recruited from a class on "Independent Psychological Research." As part of this class, $S$ s completed a number of personality questionnaires during group meetings. $S \mathrm{~s}$ scheduled individual laboratory sessions for the assessment of excitation transfer. $S \mathrm{~s}$ were told that during this session they would be asked to engage in moderate aerobic activity, and that they should wear comfortable clothing to their experimental session. $S$ s were run individually through the laboratory procedure.

\section{Personality measures}

Prior to the laboratory session, $S$ s completed a number of standard personality questionnaires in a classroom setting, including the Eysenck Personality Scales (Eysenck, Eysenck \& Barrett, 1985), the Vando Reducing/Augmenting Scale (Vando, 1974), the Need for Stimulation Scale (Herzog, Williams \& Weintraub, 1985), the Repression/Sensitization Scale (Byrne, 1964), the Affect Intensity Measure (Larsen \& Diener, 1987), and the Taylor Manifest Anxiety Scale (Taylor, 1953). These scales are all published in the professional literature and exhibit acceptable psychometric properties. These measures were selected to assess dimensions of personality that related to individual differences in arousability and affect, processes hypothesized to be related to the excitation transfer effect.

\section{Apparatus and procedure}

Upon arrival at the laboratory, $S$ s read a consent form informing them that exercise at moderate exertion would be required and inquiring about various health conditions which could be aggravated by participating in the study (i.e. heart arrhythmia, diabetes, hypertension). $S$ s with any of these conditions were not allowed to participate in the study. No $S$ s reported any medical conditions that would put them at risk during exertion and all $S$ s consented to participate in the study.

The experimenter explained to the $S$ that she would be riding a stationary bicycle ergometer as part of a study on aerobic fitness, and that the experiment session would start by collecting a baseline heart rate while the $S$ was relaxed. The experimenter then placed a photoplethysmograph on the thumb of the $S$ 's non-dominant hand and seated the $S$ in a comfortable reclining armchair. The $S$ was instructed to relax while the experimenter calibrated the polygraph.

Heart rate was monitored on a Grass Model 7D polygraph. The photoplethysmograph was attached to the $S$ 's thumb to monitor the pulse wave. Signals were routed to a Grass 7P4 cardiotachometer to detect the rising slope of each pulse wave, with the Schmitt trigger adjusted to display heart rate for each $S$ in beats per minute (BPM). Continuous output was obtained during the entire experiment by having the heart rate signal recorded on a moving strip chart. Heart rate in BPM was scored directly off the strip chart.

Once baseline measures were collected, the experimenter explained that now the $S$ was to ride on the stationary bicycle for $1 \mathrm{~min}$, maintaining a speed of $11 \mathrm{mph}$ at a tension setting sufficient to expend $245 \mathrm{~W}$ of energy. This procedure was adapted from the procedures used by Zillman, Johnson and Day (1974). Ss began to pedal slowly as the experimenter gradually increased the tension setting on the bicycle until $245 \mathrm{~W}$ and $11 \mathrm{mph}$ was achieved. A stopwatch was started and $S$ s were verbally encouraged to maintain a speed of $11 \mathrm{mph}$ as they cycled. Only one $S$ was unable to achieve and maintain this standard speed and quit peddling before the 60 -sec period ended; data from this $S$ were discarded. 
After $60 \mathrm{sec}$ of peddling the ergometer at $245 \mathrm{~W}, S \mathrm{~s}$ were helped off the ergometer and quickly seated in the armchair. At this point $S$ s were given an "activation meter" which consisted of a box with a large dial. The dial had scale readings labeled at one end with the term "Normal" and at the other end with "Highly Activated." $S$ s were told to turn the dial from "Highly Activated" to "Normal" at their own pace, as they began to feel their arousal or activation level return to what it was like when they first entered the room. The experimenter was careful to point out that $S \mathrm{~s}$ should use their breathing rate or "windedness" as the criterion for determining when they had returned to a normal level of activation.

The "Activation Meter" output a direct current electrical signal that reflected the position of the dial. This signal was routed to the polygraph and was continuously recorded on the moving strip chart alongside the $S$ 's heart rate. This technique allows the determination of: (1) peak heart rate at the end of the ergometer session, (2) the time elapsed till each $S$ felt s/he had returned to a normal level of arousal, and (3) heart rate at the time $S$ subjectively felt that had returned to normal (i.e. degree of residual arousal).

The experimenter then left the room to prepare the slides for viewing. Once the $S$ s indicated that they felt subjectively "normal," they were told they were going to view and rate 20 photographic slides. Slides were administered in 5 blocks of 4 slides each. The content of the slides varied in hecionic tone from neutral (e.g. buildings) to alternating blocks of pleasant (e.g. smiling children, couples laughing) and unpleasant tone (e.g. a terrorist bombing scene, people fighting). $S$ s viewed each slide for $10 \mathrm{sec}$ and then had a $10 \mathrm{sec}$ interval to rate the slide. They were instructed to rate how the slides made them feel by placing a slash mark anywhere along a response scale marked at one end with "extremely pleasant" and the other end with "extremely unpleasant" for each slide. After completing the rating of the slides, the plethysmograph was removed and $S$ s were debriefed and dismissed.

Six weeks later, each $S$ returned individually to the laboratory to complete several unrelated tasks. One task involved having the $S$ s rate the slides again, using the same response scale. During this session, $S$ s first completed $15 \mathrm{~min}$ of questionnaires, ensuring that $S$ s experienced the same period of relative inactivity prior to rating the slides for a second time.

\section{RESULTS}

\section{Manipulation effect on heart rate}

Before exercising on the ergometer, the mean heart rate (HR) at baseline was 81.89 BPM. Although a bit high for college students, it probably reflects some anticipatory anxiety regarding the ergometer task. After 1-min of peddling the ergometer at $245 \mathrm{~W}$, all but $3 \mathrm{Ss}$ had HRs exceeding $140 \mathrm{BPM}$, the upper limit of our cardiotachometer. After getting off the ergometer and resting in the reclining chair, the average amount of time taken to make the judgement of returning to subjectively feeling a normal level of arousal was $5.21 \mathrm{~min}$. The average HR at the time the $S$ s said they had returned to a normal level of arousal was $102.07 \mathrm{BPM}$. This value is significantly higher than the mean baseline HR $\left(t_{39}=11.42\right.$, $P<0.001$ ), suggesting that, on average, residual arousal had occured. That is, $S$ s thought they had returned to baseline even though their HRs were, on average, 20 BPM higher than baseline.

Table 1. Means and standard deviations (SD) of slide ratings" in experimental conditions

\begin{tabular}{lllll}
\hline & $\begin{array}{c}\text { Residual } \\
\text { arousal }\end{array}$ & Control & $t$ & $P$ \\
\hline Positive slides & $14.9(5.3)$ & $15.6(6.6)$ & -0.6 & NS \\
Negative slides & $44.7(5.8)$ & $43.4(7.0)$ & 1.18 & NS \\
\hline a Slide rating & scale & went from $0=$ extremely pleasant to & tondion \\
$60=$ extremely unpleasant, with a value of 30 indicating neutral \\
effective response. Hence, smaller numbers indicate more pleas- \\
ant evaluations and larger numbers indicate more unpleasant \\
evaluations.
\end{tabular}

Table 2. Correlations between personality variables and size of the excitation transfer effect

\begin{tabular}{lcc}
\hline & \multicolumn{2}{c}{ Transfer to: } \\
& Negative slides & Positive slides \\
\hline Extraversion & $-0.24^{*}$ & 0.09 \\
Neuroticism & 0.10 & $0.26^{*}$ \\
Psychoticism & -0.03 & 0.22 \\
Lie & -0.04 & 0.04 \\
Vando RAS & 0.02 & $0.44^{* *}$ \\
N-simulation & -0.04 & $0.34^{*}$ \\
Repression & -0.14 & $0.34^{*}$ \\
Anxiety & 0.16 & 0.19 \\
\hline
\end{tabular}




\section{Main effect for excitation transfer}

Table 1 presents the mean slide ratings in all experimental conditions. It can be seen that the aggregate effect is in the predicted direction, with $S$ s reporting more extreme reactions to the slides in the residual arousal condition than in the control condition. This main effect, while in the predicted direction, did not reach conventional levels of statistical significance. Nevertheless, this should not preclude an examination of individual differences, as $S \mathbf{s}$ did differ from each other in the size of the excitation transfer effect. That is, even though the overall effect is not strong, there still may be significant individual differences in the size of the excitation transfer effect.

\section{Individual differences in excitation transfer}

Table 2 reports the correlations between the personality dimensions and the arousal transfer effect to both pleasant and unpleasant stimuli. As can be seen, the extraversion and neuroticism results are consistent with the contrast effect prediction. That is, extraversion correlated with arousal transfer to negative (but not positive) stimuli, and neuroticism correlated with arousal transfer to positive (but not to negative) stimuli. The measures of sensory reducing were related to arousal transfer to positive stimuli only, as was the measure of repression. Anxiety and affect intensity did not relate to any of the arousal transfer effects.

\section{DISCUSSION}

The arousal transfer effect has been widely studied in the social psychology literature. The few attempts to examine individual differences in this effect have focused on physical characteristics, primarily cardiovascular fitness. However, because the effect involves both arousal and hedonic reactivity, it seems likely that arousal- or affect-relevant personality variables might relate to individual differences in the arousal transfer effect.

Several categories of predictions were derived from the personality literature. One set of hypotheses concerned individual differences in arousability, predicting that extraversion and sensory reducing should relate to arousal transfer, regardless of the hedonic tone of the stimulus. Another set of hypotheses was based on individual differences in hedonic sensitivity, predicting that extraversion should relate to arousal transfer to positive stimuli and neuroticism should relate to arousal transfer to negative stimuli. A third set of hypotheses made the opposite predictions, that, because extraverts are less familiar or experienced with negative affect, they should show the largest arousal transfer to unpleasant stimuli. Similarly, because high-neuroticism $S$ s are less familiar with positive affect, they should show the largest transfer effect to pleasant stimuli.

Results were generally supportive of the latter set of hypotheses. That is, Neuroticism predicted arousal transfer to pleasant (but not unpleasant) stimuli and Extraversion predicted arousal transfer to unpleasant (but not pleasant) stimuli. Ostensibly, these results are counter-intuitive, especially given the literature on personality and susceptibility to emotional states (Larsen \& Ketelaar, 1989, 1991). However, when persons are confronted with novel or unusual experiences they are more likely to seek additional sources of information. Assuming that bodily state is a source of information about one's emotional reactions, it is likely that people will attend to their bodily states when judging their emotional reactions. Moreover, it is likely that people will interrogate bodily feelings more when experiencing emotions with which they are less familiar. Because extraverts are less experienced with negative affect, their judgments about unpleasant feelings should take more effort and involve more information processing than their judgments about pleasant feelings, with which they are more familiar. Hence, residual arousal is noticed more when extraverts are judging unpleasant emotional states. Similarly, because high-neuroticism $S \mathbf{s}$ are relatively less familiar with positive emotional feelings, they should be more influenced by residual arousal when judging unpleasant feelings, with which they are more familiar. Theoretically, people will take account of residual arousal when assessing feeling states with which they have relatively less experience.

Besides the substantive findings, this study illustrates an approach to experimental personality research. Whenever an experimental paradigm (in this case, excitation transfer) is based on a construct related to traditional personality dimensions (in this case, arousability and emotional 
reactivity), then that experimental paradigm may be used to examine individual difference effects. In this study, predictions were made based on individual differences in arousability and emotional reactivity, constructs presumed to underlie the excitation transfer effect. Many other experimental paradigms might be fruitfully employed by personality researchers, such as the dissonance paradigm or the audience facilitation effect, both of which are assumed to be based on the construct of arousal. Bridges between such "main effect" paradigms and personality theory may be constructed on the basis of shared underlying constructs. If an experimental effect is based on a construct along which people differ (i.e. arousability), then the experimental paradigm may be useful for studying the implications of personality differences. For example, if the audience facilitation effect works on the basis of increasing arousal, then the experimental effects of audience manipulations should be different for introverts and extraverts. Many other bridging constructs, such as anxiety, reward motivation, or sensory sensitivity, might be fruitfully employed to connect personality theories to 'main-effect' paradigms, thereby increasing our knowledge of both.

Acknowledgements - Preparation of this manuscript was supported, in part, by Research Scientist Development Award KO1-MH00704 and Grant RO1-MH42057 from the National Institute of Mental Health to Randy J. Larsen.

\section{REFERENCES}

Byrne, D. (1964). Repression-sensitization as a dimension of personality. Progress in Experimental Personality Psychology, $1,169-220$.

Cantor, J. R., Zillman, D. \& Day, K. D. (1978). Relationship between cardiorcspiratory fitncss and physiological responses to films. Perceptual and Motor Skills, 46, 1123-1130.

Costa, P. T. Jr \& McCrae, R. R. (1980). Influence of extraversion and neuroticism on subjective well-being: Happy and unhappy people. Journal of Personality and Social Psychology, 38, 668-678.

Cox, J. P., Evans, J. F. \& Jamieson, J. L. (1979). Aerobic power and tonic heart rate responses to psychosocial stressors. Personality and Social Psychology Bulletin, 5, 160-163.

Donnerstein, E. \& Hallam, J. (1978). Facilitating effects of erotica on aggression against women. Journal of Personality and Social Psychology, 36, 1270-1277.

Emmons, R. A. \& Diener, E. (1985). Personality correlates of subjective well-being. Personality and Social Psychology Bulletin, 11, 89-97.

Eysenck, H. J. (1967). The biological basis of personality. Springfield, IL: Charles Thomas.

Eysenck, S. B. G., Eysenck, H. J. and Barrett, P. (1985). A revised version of the Psychoticism scale. Personality and Individual Differences, 6, 21-29.

Gray, J. A. (1964). Pavlov's typology. New York: McMillon.

Gray, J. A. (1970). The psychophysiological basis of introversion-extraversion. Behavior Research and Therapy, 8, $249-266$.

Gray, J. A. (1987). The neuropsychology of emotion and personality structure. Zhurnal Vysshei Nervnoi Deyatel'nosti, 37, $1011-1024$.

Herzog, T. R., Williams, D. M. \& Weintraub, D. J. (1985). Meanwhile, back at personality ranch: The augmenters and reducers ride again. Journal of Personality and Social Psychology, 48, 1342-1352.

Jaffe, Y. (1981). Sexual stimulation: Effects on prosocial behavior. Psychological Reports, 48, 75-81.

Larsen, R. J. \& Diener, E. (1987). Affect intensity as an individual difference characteristic: A review. Journal of Research in Personality, 21, 1-39.

Larsen, R. J. \& Ketelaar, T. (1989). Extraversion, neuroticism and susceptibility to positive and negative mood induction procedures. Personality and Individual Differences, 10, 1221-1228.

Larsen, R. J. \& Ketelaar, T. (1991). Personality and susceptibility to positive and negative emotional states. Journal of Personality and Social Psychology, 61, 132-140.

Mueller, C. W. \& Donnerstein, E. (1981). Film-facilitated arousal and prosocial behavior. Journal of Experimental Social Psychology, 17, 31-41.

O'Malley, M. N. \& Gillette, C. S. (1984). Exploring the relations between traits and emotions. Journal of Personality, 52, 274-284.

Pennebaker, J. (1978). The psychology of physical symptoms. New York: Springer-Verlag.

Petrie, A. (1967). Individuality in pain and suffering. Chicago, IL: Chicago University Press.

Taylor, J. (1953). A personality scale of manifest anxiety. Journal of Abnormal and Social Psychology, 48, 285-290.

Vando, A. A. (1974). The development of the R-A scale: A paper-and-pencil measure of pain tolerance. Personality and Social Psychology Bulletin, 1, 28-29.

Warr, P. B., Barter, J. \& Brownbridge, G. (1983). On the independence of positive and negative affect. Journal of Personality and Social Psychology, 44, 644-651.

Watson, D. \& Pennebaker, J. W. (1989). Health complaints, stress, and distress: Exploring the central role of negative affectivity. Psychological Review, 96, 234-254.

Zillman, D. (1971). Excitation transfer in communication-mediated aggressive behavior. Journal of Experimental Social Psychology, 7, 419-434.

Zillman, D. (1978). Attributions and misattributions of excitatory reactions. In Harvey, J. H., Ickes, W. \& Kidd, R. F. (Eds), New directions in attribution research (Vol. 2). Hillsdale, NJ: Erlbaum.

Zillman, D. (1979). Hostility and aggression. Hillsdale, NJ: Erlbaum.

Zillman, D. (1983). Arousal and aggression. In Geen, R. G. \& Donnerstein, E. (Eds), Aggression: Theoretical and empirical reviews (Vol. 1). New York: Academic. 
Zillman, D. \& Bryant, J. (1974). Effect of residual excitation on the emotional response to provocation and delayed aggressive behavior. Journal of Personality and Social Psychology, 30, 782-791.

Zillman, D., Johnson, R. C. \& Day, K. D. (1974). Attribution of apparent arousal and proficiency of recovery from sympathetic activation affecting excitation transfer to aggressive behavior. Journal of Experimental Social Psychology, $10,503-515$.

Zillman, D., Katcher, A. H. \& Milavsky, B. (1972). Excitation transfer from physical exercise to subsequent aggressive behavior. Journal of Experimental Social Psychology, 8, 247-259.

Zillman, D., Bryant, J., Comisky, P. W. \& Medoff, N. J. (1981). Excitation and hedonic valence in the effect of erotica on motivated intermale aggressive behavior. European Journal of Social Psychology, 11, 233-252. 